\title{
Investigation of key autophagy-and mitophagy-related proteins and gene expression in BALF cells from patients with IPF and RA-ILD
}

\author{
EIRINI VASARMIDI ${ }^{1,2^{*}}$, STELLA SARANTOULAKI ${ }^{1 *}$, ATHINA TRACHALAKI ${ }^{1}$, GEORGE MARGARITOPOULOS $^{3}$, \\ ELENI BIBAKI $^{1,2}$, DEMETRIOS A. SPANDIDOS ${ }^{4}$, NIKOLAOS TZANAKIS ${ }^{2}$ and KATERINAANTONIOU ${ }^{1,2}$ \\ ${ }^{1}$ Laboratory of Molecular and Cellular Pneumonology, Medical School, University of Crete; \\ ${ }^{2}$ Department of Thoracic Medicine, University Hospital of Heraklion, 71110 Heraklion, Crete, Greece; \\ ${ }^{3}$ Interstitial Lung Disease Unit, Royal Brompton and Harefield NHS Foundation Trust, London, UK; \\ ${ }^{4}$ Laboratory of Clinical Virology, University of Crete, 71110 Heraklion, Crete, Greece
}

Received May 31, 2018; Accepted August 1, 2018

DOI: $10.3892 / \mathrm{mmr} .2018 .9356$

\begin{abstract}
Idiopathic pulmonary fibrosis (IPF) is a chronic and irreversible interstitial lung disease with a poor prognosis and limited therapeutic options. Over the past decade, research efforts have focused on the pathogenetic mechanisms involved in this enigmatic lung disease. Rheumatoid arthritis (RA) is a chronic inflammatory autoimmune disease often complicated by the development of interstitial lung disease (ILD), leading to high mortality and morbidity. Autophagy is a process regulating the turnover of subcellular components and organelles, and represents a major cellular homeostatic mechanism. Recent evidence suggests a role of autophagy and mitochondrial dysfunction in the development of IPF, focusing on lung fibroblasts and epithelial cells. The aim of this study was to examine the mRNA levels of molecules involved inthe autophagy pathway in bronchoalveolar lavage fluid (BALF)-derived cellsfrom patients with IPF in comparison topatients with RA demonstrating lung involvement (ILD) by RT-qPCR. The significant upregulation of BECLIN1 was observed in patients with RA-ILD compared with those with
\end{abstract}

Correspondence to: Professor Katerina M. Antoniou, Laboratory of Molecular and Cellular Pneumonology, Medical School, University of Crete, Voutes, 71110 Heraklion, Crete, Greece

E-mail: kantoniou@med.uoc.gr

*Contributed equally

Abbreviations: IPF, idiopathic pulmonary fibrosis; RA, rheumatoid arthritis; RA-ILD, rheumatoid arthritis-interstitial lung disease; HRCT, high resolution computed tomography; COPD, chronic obstructive pulmonary disease; BALF, bronchoalveolar lavage fluid; AECs, alveolar epithelial cells; RA-FLS, rheumatoid arthritis fibroblast-like synoviocytes

Key words: alveolar, macrophage, autophagy, idiopathic pulmonary fibrosis, rheumatoid arthritis, interstitial lung disease
IPF. Other genes involved in the autophagy pathway were also examined, such as Unc-51 like autophagy activating kinase 1 (ULK1), BCL2 interacting protein 3 (BNIP3) and p62. No differences in the mRNA expression levels of these genes were observed. As regards the selective degradation of mitochondria and mitophagy, similar PTEN-induced putative kinase 1 (PINK1) and PARKIN; E3 ubiquitin ligase (PRKN) expression, as well as PINK1 protein levels, were observed. On the whole, the findings of this study demonstrate an increased expression of BECLIN1 in BALF cells from patients with RA-ILD compared with those from patients with IPF, while similar levels in other key molecules implicating in the autophagy pathway were observed in patients with IPF and RA-ILD.

\section{Introduction}

Autophagy is a membrane-dependent mechanism for the turnover of subcellular components and represents a major cellular homeostatic mechanism. It serves as a source of metabolic fuel, it removes aggregated proteins or dysfunctional mitochondria, and determines cell fate (1). In addition to its basic role in the turnover of proteins and organelles, autophagy has multiple physiological and pathophysiological functions, including roles in cell differentiation and immune response (2). Changes in autophagy affect the removal of aged and defective mitochondria, protein aggregates and internalized bacteria. The function of autophagy in human disease appears to be pleiotropic, with implications in cancer, metabolic, neurodegenerative, cardiovascular diseases, autoimmune, as well as lung diseases (2,3). More specifically, in the lungs, enhanced autophagy has been described in epithelial cells from patients with chronic obstructive pulmonary disease (COPD), leading to apoptosis $(4,5)$. By contrast, the alveolar macrophages of patients with COPD present an impairment in autophagy (6).

Idiopathic pulmonary fibrosis (IPF) is a chronic progressive fibrotic disease of unknown etiology (7). The current dogma in the disease pathogenesis centers on lung epithelial cell dysfunction in an aging lung, which leads to fibroblast 
activation and excessive extracellular matrix deposition, finally leading to the destruction of the lung architecture. IPF shares some intriguing similarities with rheumatoid lung disease, in pathogenesis $(8,9)$, as well as disease progression (10). Rheumatoid arthritis (RA) is an autoimmune disease, characterized by joint inflammation and is thought to be driven by antibodies against citrullinated proteins (11). Interstitial lung disease (ILD) commonly complicates RA, in up to $60 \%$ in certain studies, and frequently precedes joint inflammation (12). Smoking, a common risk factor in both idiopathic- and autoimmune-driven lung disease, is associated with changes in the autophagy pathway. The accumulation of autophagosomes is present in lung tissue from patients with COPD (5) and in lung epithelial cells exposed to cigarette smoke extract (13).

Aging, a hallmark of IPF pathogenesis, is also associated with insufficient autophagy (14), while increasing evidence suggests that the autophagic process is impaired in $\operatorname{IPF}(15,16)$. By contrast, in RA, increased autophagy has been linked with the enhanced survival of RA fibroblast-like synoviocytes (RA-FLS) (17), a major source of pro-inflammatory cytokines. Intriguingly, the induction of autophagy in antigenpresenting cells can lead to citrullinated peptide presentation, a hallmark of RA pathogenesis (18). In alveolar macrophages, citrullinated peptides are increased in anti-CCP-positive RA patients, as well as in IPF (19). Thereafter, a contradicting role of autophagy in both diseases has been proposed and it is likely that the role of autophagy is cell-specific, as previous studies on COPD have suggested $(5,6,13)$.

Alveolar macrophages are essential for maintaining the sterility of the lung (20) and have been proposed to play a role in lung fibrosis (21), both in initiating the inflammatory response after injury, as well as in resolution and repair. As regards the autophagy process in macrophages in fibrosis, little is known. Of note, a study by Drakopanagiotakis et al identified that alveolar macrophages of patients with IPF had a decreased apoptotic rate, which may enhance the progression of IPF (22), while AKT1 has been shown to mediate apoptosis resistance by enhancing mitophagy (23).

Therefore, the purpose of the current study was to examine the levels of the major regulatory molecules of the autophagic pathway in bronchoalveolar lavage fluid (BALF) cells derived from patients with IPF and RA-ILD. Although RA is an autoimmune disease mainly associated with inflammatory pathways and is characterized by a different disease progression and therapeutic strategies, it is known that lung involvement in RA leading to ILD and destruction of the lung parenchyma, finally results in the same survival as IPF. Thus, apart from obvious differences, these two diseases present some rather intriguing similarities at the clinical and molecular level $(24,25)$. It is of great interest to investigate the molecular pathways and potent pathogenetic mechanisms, not only by focusing on systemic markers or joint inflammation, but also by evaluating lung microenvironment.

\section{Materials and methods}

Patients. A total of 75 subjects were recruited from the Department of Thoracic Medicine, University Hospital of Heraklion, Greece, consisting of patients with IPF $(n=55)$
Table I. The sequences of the primers used.

\begin{tabular}{ll}
\hline Gene name & \multicolumn{1}{c}{ Primer sequences } \\
\hline$p 62$ & F: agctgccttgtacccacatc \\
& R: cagagaagcccatggacag \\
BECLIN1 & F: tcaccatccaggaactcaca \\
& R: tggctcctctcctgagttagtc \\
ULK1 & F: gccetcgtacccaagctc \\
& R: gaggccagggtcttctgc \\
BNIP3 & F: tgctgctctctcatttgctg \\
& R: gactccagttcttcatcaaaaggt \\
PINK1 & F: ggagtatggagcagtcacttacag \\
& R: ggcagcacatcagggtagtc \\
PARKIN & F: cacctacccagtgaccatga \\
& R: cgacctccactgggaaac \\
GAPDH & F: agccacatcgctcagacac \\
& R: gcccaatacgaccaaatcc
\end{tabular}

and patients with RA-ILD ( $\mathrm{n}=20)$ between March, 2014 to March, 2017.

The diagnosis of IPF was based on European Respiratory Society (ERS)/American Thoracic Society (ATS) clinical guidelines and high resolution computed tomography (HRCT) criteria or on open or video-assisted thoracoscopic biopsy $(\mathrm{n}=5)$, with all biopsies reviewed by the same two histopathologists (26). In accordance with the aforementioned criteria, any patient presenting any known cause of pulmonary fibrosis, such as a systemic connective tissue disorder, was excluded from this study using both immunologic screening and rheumatologic clinical evaluation (26). All patients with IPF were newly diagnosed and had not received any previous treatment. The criteria for the diagnosis of connective tissue disease CTD included the American College of Rheumatology (ACR) 1987 revised criteria for the classification of RA (27). Patients with RA-ILD had HRCT findings indicative of definite ILD. Informed consent was obtained from all patients who participated in this study. This study was approved by the Ethics Committees of the University Hospital of Heraklion (Crete, Greece; IRB no. 17030)

$B A L F$ processing. BALF was obtained from all patients at room temperature. Briefly, a flexible bronchoscope was wedged into a sub-segmental bronchus of a predetermined region of interest based on radiographical findings. A BAL technique was performed by instilling a total of $180 \mathrm{ml}$ of normal saline in $60-\mathrm{ml}$ aliquots, each retrieved by low suction. Samples were filtered through sterile 70-nm cell strainers (BD Biosciences, San Jose, CA, USA) and centrifuged at $500 \mathrm{xg}$ or $5 \mathrm{~min}$ at $4^{\circ} \mathrm{C}$. Cell pellets were washed and re-suspended with cold PBS. Total cell count and cell viability were subsequently assessed using Trypan blue (ICN Pharmaceuticals, Costa Mesa, CA, USA). A total of 1-1.5 million cells were centrifuged and cell pellets were homogenised in TriReagent ${ }^{\mathrm{TM}}$ (MBL) for total RNA, or RIPA buffer (Invitrogen/Thermo Fisher Scientific, Waltham, MA, USA) containing protease and phosphatase inhibitors; 
Table II. Patient characteristics.

\begin{tabular}{lccc}
\hline Characteristics & IPF & RA-ILD & P-value \\
\hline Number & 55 & 20 & \\
Sex (male/female) & $47 / 8$ & $7 / 13$ & $<0.0001$ \\
Packyears & $15(0-150)$ & $0(0-175)$ & $\mathrm{NS}$ \\
Non-smokers & 16 & 10 & $\mathrm{NS}$ \\
Ex smokers & 30 & 4 & 0.02 \\
Current smokers & 5 & 3 & $\mathrm{NS}$ \\
Age (years) & $68(27-86)$ & $67.50(46-81)$ & $\mathrm{NS}$ \\
\hline
\end{tabular}

Data are presented as the median (range). The Chi-squared test and Mann-Whitney U test were used as appropriate considering $\mathrm{P}<0.05$ as statistically significant. NS, not significant; RA-ILD, rheumatoid arthritis-interstitial lung disease; IPF, idiopathic pulmonary fibrosis.

Pierce/Thermo Fisher Scientific) for SDS-PAGE/western blot analysis, followed by storage at $-80^{\circ} \mathrm{C}$. Differential cell population count was analysed by May-Grunewald-Giemsa staining as previously described (19).

RNA extraction and mRNA expression analysis. Total RNA was isolated from BALF cells using the mirVana ${ }^{\mathrm{TM}}$ miRNA isolation kit (Ambion/Thermo Fisher Scientific) with minor modifications. The quality and quantity of the isolated RNA was assessed by agarose gel electrophoresis and spectrophotometry (NanoDrop/Thermo Fisher Scientific), respectively. For gene expression analyses, $500 \mathrm{ng}$ of total RNA were first treated with DNA-free (Ambion/Thermo Fisher Scientific) in order to remove genomic DNA contamination, followed by 1st strand cDNA synthesis using Maxima $\mathrm{RT}^{\mathrm{TM}}$ and real-time qPCR analysis using Maxima SYBR-Green pPCRmix (both from Fermentas/Thermo Fisher Scientific) on a Mx3005P qPCR system (Agilent Technologies, Santa Clara, CA, USA). The thermocycling conditions were as follows: $95^{\circ} \mathrm{C}$ for $5 \mathrm{~min}$, 40 cycles of $95^{\circ} \mathrm{C}$ for $20 \mathrm{sec}, 55^{\circ} \mathrm{C}$ for $20 \mathrm{sec}$ and $72^{\circ} \mathrm{C}$ for $20 \mathrm{sec}$, followed by melting curve with a ramp speed of $1^{\circ} \mathrm{C}$ per sec between $60^{\circ} \mathrm{C}$ and $95^{\circ} \mathrm{C}$. The probe and primer sequences are summarized in Table I. GAPDH levels were used as endogenous control for the normalization of mRNA expression levels in BALF samples. Relative expression values for the patient cohort were calculated by the following equation: Relative gene expression $=$ Effgoi ${ }^{\text {(Calibrator Ctgoi }- \text { SampleCtgoi) }} /$ Effref $^{\text {(Calibrator Ctref }- \text { SampleCtref) }}$, where (Eff refers to efficiency, goi to gene of interest, and ref to reference gene).

Western blot analysis. Total protein lysates (20 ng) of BALF samples were lysed in RIPA buffer including protease inhibitor (both from Thermo Fisher Scientific). Total protein mass was determined by Coomassie (Bradford protein assay kit) (Thermo Fisher Scientific). The proteins were separated by $12 \%$ SDS-PAGE and transferred onto $0.45 \mathrm{~nm}$ nitrocellulose membranes (Bio-Rad, Hercules, CA, USA), followed by the detection of p62 with an anti-p62 mouse monoclonal antibody, at a 1/500 dilution (M162-3; MBL, Woburn, MA, USA), and $\beta$-actin using an anti- $\beta$-actin mouse monoclonal antibody at a 1/5,000 dilution (A2228; Sigma, St. Louis, MO, USA).

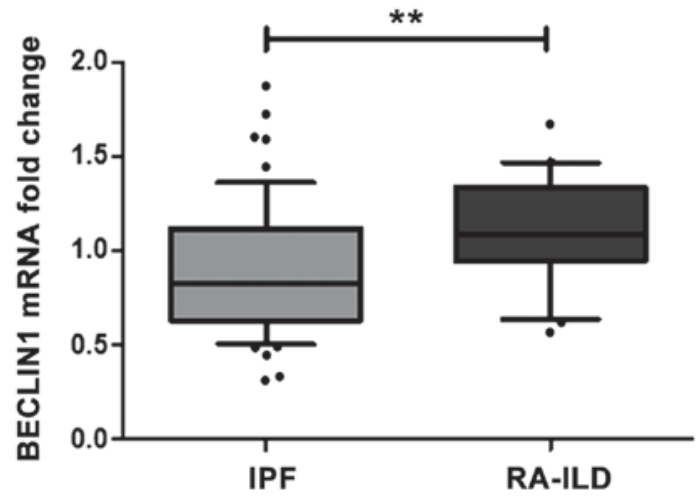

Figure 1. RT-PCR analysis revealed the upregulation of BECLIN1 in patients with RA-ILD $(n=20)$ when compared to those with IPF $(n=55)$ samples. Data were normalized to GAPDH and are represented as the median with $10-90 \%$ range. ${ }^{* *} \mathrm{P}$-value $=0.0089$, determined by the Mann-Whitney U test. RA-ILD, rheumatoid arthritis-interstitial lung disease; IPF, idiopathic pulmonary fibrosis.

Appropriate goat anti-mouse HRP conjugated secondary antibody at a 1/2,000 dilution (AP124P; Chemicon/Merck KGaA, Darmstadt, Germany) was used and immunodetection was performed with enhanced chemiluminescence reagent Luminata $^{\mathrm{TM}}$ (Millipore, Billerica, MA, USA). Bands were visualised with the ChemiDoc XRS+ system and densitometric analyses were performed using Image $\mathrm{Lab}^{\mathrm{TM}}$ software (both from Bio-Rad).

Statistical analysis. Gene expression analysis was performed using Prism 5 software following the incorporation of relative expression values in average (duplicates) normalized to GAPDH. Group comparisons were made using the Mann-Whitney U test or the Chi-square testing as appropriate. A P-value $<0.05$ was considered to indicate a statistically significant difference.

\section{Results}

Patient characteristics. The data of the patients, including those in the IPF and RA-ILD group used in this study are summarized in Table II.

Increased expression of BECLIN1 in BALF cells derived from patients with RA-ILD compared with those from patients with IPF. The initiation of autophagosome formation requires specific regulatory complexes, including two major contributors, BECLIN1 and Unc-51 like autophagy activating kinase $1(U L K 1)$. The expression levels of these two essential molecules were examined in BALF cells derived from patients with IPF and RA-ILD. Statistically significant higher mRNA levels of BECLIN1 were observed in the BALF cells from patients with RA-ILD in comparison to those from patients with IPF (Fig. 1), while similar levels of $U L K 1$ were detected (Table III).

No differences in the MRNA and protein levels of the adaptor molecule p62 in BALF cells of IPF and RA-ILD patients. A major role in the selective degradation of components or organelles is played by the adaptor molecules, mainly including p62 (also known as sequestosome1) and BCL2 interacting protein 3 (BNIP3). Although the adaptor molecules are not 


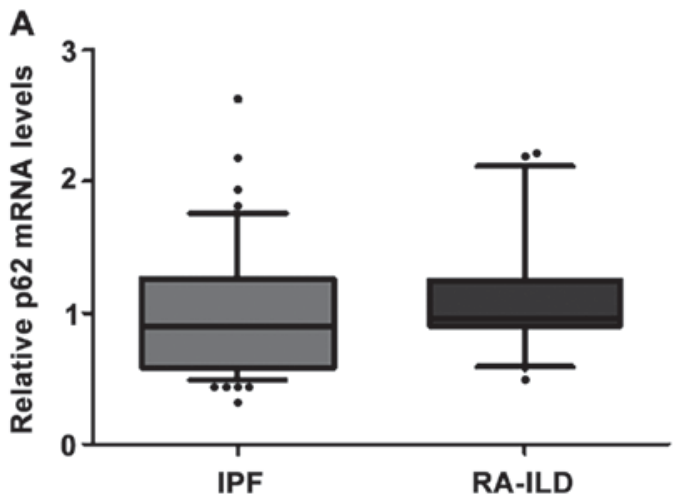

B

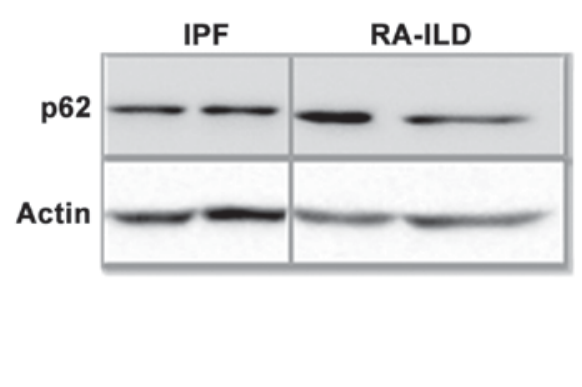

Figure 2. (A) mRNA expression of $p 62$ in patients with IPF $(\mathrm{n}=55)$ and RA-ILD $(\mathrm{n}=20)$. Data were analyzed by RT-qPCR and normalized to GAPDH. No significant differences, as determined by the Mann-Whitney $U$ test $(P>0.05)$. Data are represented as the median with $10-90 \%$ range. (B) Representative western blot showing similar protein levels of p62 in BALF cells from patients with IPF and RA-ILD. Analysis of patients with IPF ( $n=5)$ and RA-ILD ( $n=3$ ) revealed no significant differences in the p62 protein levels (p62/actin). RA-ILD, rheumatoid arthritis-interstitial lung disease; IPF, idiopathic pulmonary fibrosis; BALF, bronchoalveolar lavage fluid.


Figure 3. Measurement of (A) PINK1 mRNA, (B) PARKIN mRNA and (C) PINK1 protein levels revealed similar results in BALF cells derived from patients with IPF and RA-ILD. (A and B) PINK1 and PARKIN expression levels showed no statistically significant differences between the IPF and RA-ILD samples. Data were normalized to GAPDH and are represented as the median with 10-90\% range. No significant differences, as determined by the Mann-Whitney $\mathrm{U}$ test $(\mathrm{P}>0.05)$. (C) Western blot analysis of pink1 protein normalized to actin. No significant difference, as determined by the Mann-Whitney $\mathrm{U}$ test $(\mathrm{P}>0.05)$. RA-ILD, rheumatoid arthritis-interstitial lung disease; IPF, idiopathic pulmonary fibrosis; BALF, bronchoalveolar lavage fluid.

Table III. Expression levels of the genes analyzed.

\begin{tabular}{lllc}
\hline Gene & \multicolumn{1}{c}{ IPF } & \multicolumn{1}{c}{ RA-ILD } & P-value \\
\hline p62 & $0.90(0.32-5.47)$ & $0.95(0.49-2.22)$ & NS \\
BNIP3 & $0.98(0.16-10.37)$ & $1.07(0-14.29)$ & NS \\
BECLIN1 & $0.83(0.32-1.88)$ & $1.09(0.56-1.66)$ & $<0.01$ \\
ULK1 & $1.24(0.40-3.59)$ & $1.09(0-8.76)$ & NS \\
S100A9 & $0.70(0.28-5.71)$ & $0.81(0.35-3.22)$ & NS \\
PINK1 & $0.99(0.26-2.33)$ & $0.83(0.12-2.68)$ & NS \\
PARKIN & $0.99(0-3.64)$ & $0.88(0-1.68)$ & NS \\
\hline
\end{tabular}

$\operatorname{IPF}(\mathrm{n}=55)$, RA-ILD ( $\mathrm{n}=20)$. Data are presented as the median (range). Mann-Whitney $\mathrm{U}$ test, $\mathrm{P}<0.05$ is considered statistically significant. RA-ILD, rheumatoid arthritis-interstitial lung disease; IPF, idiopathic pulmonary fibrosis. required for autophagosome formation, they were evaluated in order to assess the levels of autophagy. The mRNA levels of p62 (Fig. 2A) and BNIP3 (Table III) were measured in BALF cells and no significant difference was observed between the IPF and RA-ILD groups. Moreover, the measurement of the p62 protein levels is a widely used indicator of degradation through autophagy machinery $(2,28)$. Thus, the protein levels of p62 in fresh BALF cells of a subgroup of patients were also analyzed and they were found similar in the IPF and RA-ILD samples (Fig. 2B).

Evaluation of markers of mitochondrial homeostasis. The role of impaired mitochondrial recycling has been highlighted in aging lung and in pulmonary disorders including IPF $(14,29,30)$. Mitochondrial dysfunction due to insufficient autophagy has been shown in alveolar epithelial cells (AEC) IIs and fibroblasts of IPF patients (30-32). The two mostly 
investigated molecules that determine mitochondrial degradation are PTEN-induced putative kinase 1 (PINK1) and $P A R K I N$. No differences in the expression levels of PINK1 and PARKIN were observed between the IPF $(\mathrm{n}=55)$ and RA-ILD $(\mathrm{n}=20)$ groups (Fig. 3A and B). The PINK1 protein levels were also evaluated in the IPF $(n=11)$ and RA-ILD $(n=7)$ BALF cells and no significant difference was observed (MannWhitney U test, no significance). A representative western blot is presented in Fig. 3C.

\section{Discussion}

Idiopathic- and autoimmune-related ILD share some intriguing similarities in clinical presentation $(10,33)$, as well as in pathogenetic processes $(19,34)$ and genetic predisposition $(35)$. In the current study, gene and protein expression of key autophagyrelated molecules were evaluated in BALF cells derived from patients with IPF and RA-ILD. BECLIN1 expression was significantly upregulated in the BALF cells derived from patients with RA-ILD compared to those derived from patients with IPF. However, additional gene expression analysis of $p 62$, $U L K 1$ and BNIP3, also involved in the autophagy pathway, did not display any statistically significant differences between the groups. As regards the selective degradation of mitochondria by mitophagy, we found the same mRNA expression levels of PINK1 and PARKIN, while no significant differences were observed in the protein levels of PINK1.

Oxidative stress, endoplasmic reticulum stress and hypoxia are not only mechanisms implicated in the pathogenesis of IPF, but also inducers of autophagy (29).A previous study by Patel et al examined markers of autophagic activity (LC3 and p62) in human IPF lungs and the number of autophagosomes and it was detected that autophagy was not induced in human IPF lungs (15). Another study claimed that there was insufficient autophagy in alveolar epithelial cells in lungs of patients with IPF, which may lead to epithelial cell senescence, whereas in fibroblasts, insufficient autophagy may induce differentiation into myofibroblasts (36). Bueno et al proposed that there may be an induction of the autophagy process in alveolar epithelial cells (AECs) in IPF, but finally the autophagy flux is impaired (31). Contrariwise, in RA, increased autophagy has been associated with RA fibroblast-like synoviocytes (RA-FLS) (37), leading to the production of citrullinated proteins, a hallmark in disease pathogenesis (38). Of note, one commonly used medication for RA treatment is hydroxychloroquine (Plaquenil), a disease-modifying anti-rheumatic agent. Notably, hydroxychloroquine is an inhibitor of lysosomal acidification and mostly accumulates in acidic cytoplasmic vesicles. Another study demonstrated that the inhibition of the autophagy-related protein, histone deacetylase 6 (HDAC6), reduces the inflammatory cytokine secretion from macrophages and FLS, and ameliorates arthritis disease severity in mouse models (39). Alterations to the regulation of autophagy have also been identified to contribute to the progression of other autoimmune diseases (40).

Although autophagy has been well described in IPF AECs and fibroblasts and in RA sunovium, its role in alveolar macrophages remains unknown. Alveolar macrophages represent very important cells in lung composition, since they play a central role in inflammation, host defense and tissue homeostasis. They participate in all stages of the fibrotic process, as they serve as key regulators of fibroblast recruitment, proliferation and activation. It is currently believed that macrophages within the lung orchestrate the downstream progression and maintenance of fibrosis (13). Furthermore, smoking, related to the pathogenesis of both diseases, has been linked to a defect in functional autophagy in alveolar macrophages (6). BAL is a minimally invasive procedure, providing immune cells from the alveolar compartment and is an excellent research tool for the investigation of alveolar macrophages. This study demonstrated that, in BALF cells, the mRNA levels of major contributors to the selective degradation of cellular components; $p 62, B N I P 3$ and $U L K 1$, were similarly expressed in idiopathic and autoimmune lung fibrosis.

Of note, BECLIN1, a major autophagic regulator and tumor suppressor protein (41), was found significantly upregulated in BALF cells derived from patients with RA-ILD compared to those from patients with IPF. BECLIN1 is required for the initiation of autophagosome formation. Hypoxia, a critical component in interstitial lung diseases $(42,43)$ regulates autophagy, and this activation requires BECLIN1 (44). Recent data have indicated that BECLINI is downregulated in fibroblasts from patients with IPF (45). Furthermore, Nintedanib, an approved medication for the treatment of IPF, has been demonstrated to induce autophagy in IPF fibroblasts in a BECLIN1-dependent manner (16). In this study, although the mRNA levels of BECLIN1 were significantly lower in BALF cells from patients with IPF compared to those from patients with RA-ILD, this is not a sufficient marker alone to imply the decrease in the regulation of autophagy in IPF.

Mitophagy is a form of macroautophagy that selectively degrades damaged mitochondria. Mitochondrial kinase PINK1 senses damage and signals this to the cytosolic E3 ligase, PARKIN. PINK1 expression decreases with age, leading to the accumulation of damaged mitochondria. In lungs affected by IPF, further dysregulation of many of the regulatory mechanisms that control mitochondrial function has recently been identified in epithelial cells, fibroblasts and macrophages (30). Dysfunctional mitochondria in IPF have been associated with the decreased expression of the major regulatory molecule of mitophagy, PINK1, in AECs from patients with IPF (31); however, of note, no difference was observed in fibroblasts (31). PINK1-deficient mice exhibit susceptibility to apoptosis and spontaneous TGF $\beta$-driven lung fibrosis and a low expression of PINK1 promotes fibroblast to myofibroblast transition (46). Similarly, PARKIN deficiency enhanced myofibroblast differentiation and pro-fibrotic signaling (32). Notably, in this study, we observed that, PINK1 and PARKIN were similarly expressed in BALF cells from patients with IPF and RA-ILD, which suggests a role of mitophagy in rheumatoid lung disease.

Although the results of this study provide evidence of similar levels of major autophagy molecules in BALF cells from patients with IPF and RA-ILD, certain limitations should be noted. Autophagy is a dynamic process and the evaluation of the expression of key molecules in the process, is not an efficient marker of the autophagic levels. A better characterization of the autophagic pathway in interstitial lung disease requires the evaluation not only of the mRNA levels of certain regulatory molecules and the protein levels at a steady state condition, but also an assessment of the autophagic flux (28). 
For instance, using a substance that can block the turnover of autophagosomes is a widely used method to estimate the potent accumulation of autophagosomes.

Conclusively, the findings of this study provide insight into the autophagic pathway in BALF cells derived from patients with both idiopathic- and autoimmune-related ILD. Further research is required to better define the autophagy machinery in BALF cells from patients with ILD, with regards to understanding the disease pathogenesis and establishing novel therapeutic targets.

\section{Acknowledgements}

Not applicable.

\section{Funding}

This study was funded by an annual research grand of the Hellenic Thoracic Society HTS-2013.

\section{Availability of data and materials}

The datasets used and/or analyzed during the current study are available from the corresponding author on reasonable request.

\section{Authors' contributions}

EV and SS performed the experiments and analyzed and interpreted the data. EB, AT and GM also contributed to the acquisition of human samples and interpretation of the data. EV and AT wrote the manuscript. DAS, NT and KA made substantial contributions to the conception and design of this study. All authors were involved in the drafting of the manuscript, and all have read and approved the final version of the manuscript.

\section{Ethics approval and consent to participate}

Informed consent was obtained from all patients who participated in this study. This study was approved by the Ethics Committees of the University Hospital of Heraklion (Crete, Greece; IRB no. 17030).

\section{Patient consent for publication}

Not applicable.

\section{Competing interests}

DAS is the Editor-in-Chief for the journal, but had no personal involvement in the reviewing process, or any influence in terms of adjudicating on the final decision, for this article.

\section{References}

1. Cuervo AM: Autophagy and aging: Keeping that old broom working. Trends Genet 24: 604-612, 2008.

2. Deretic V, Saitoh T and Akira S: Autophagy in infection, inflammation and immunity. Nat Rev Immunol 13: 722-737, 2013.

3. Nakahira K, Pabon Porras MA and Choi AM: Autophagy in pulmonary diseases. Am J Respir Crit Care Med 194: 1196-1207, 2016.
4. Hwang JW, Chung S, Sundar IK, Yao H, Arunachalam G, McBurney MW and Rahman I: Cigarette smoke-induced autophagy is regulated by SIRT1-PARP-1-dependent mechanism: Implication in pathogenesis of COPD. Arch Biochem Biophys 500: 203-209, 2010.

5. Chen ZH, Kim HP, Sciurba FC, Lee SJ, Feghali-Bostwick C, Stolz DB, Dhir R, Landreneau RJ, Schuchert MJ, Yousem SA, et al: Egr-1 regulates autophagy in cigarette smoke-induced chronic obstructive pulmonary disease. PLoS One 3: e3316, 2008.

6. Monick MM, Powers LS, Walters K, Lovan N, Zhang M, Gerke A, Hansdottir S and Hunninghake GW: Identification of an autophagy defect in smokers' alveolar macrophages. J Immunol 185: 5425-5435, 2010.

7. Wolters PJ, Collard HR and Jones KD: Pathogenesis of idiopathic pulmonary fibrosis. Annu Rev Pathol 9: 157-179, 2014.

8. Johnson C, Giles JT, Bathon J, Lederer D, Hoffman EA, Barr RG and Danoff SK: Smoking and Subclinical ILD in RA versus the Multi-Ethnic Study of Atherosclerosis. PLoS One 11: e0153024, 2016.

9. Bernstein EJ, Barr RG, Austin JHM, Kawut SM, Raghu G, Sell JL, Hoffman EA, Newell JD Jr, Watts JR Jr, Nath PH, et al: Rheumatoid arthritis-associated autoantibodies and subclinical interstitial lung disease: The Multi-Ethnic Study of Atherosclerosis. Thorax 71: 1082-1090, 2016.

10. Solomon JJ, Chung JH, Cosgrove GP, Demoruelle MK, Fernandez-Perez ER, Fischer A, Frankel SK, Hobbs SB, Huie TJ, Ketzer J, et al: Predictors of mortality in rheumatoid arthritisassociated interstitial lung disease. Eur Respir J 47: 588-596, 2016.

11. Luban S and Li ZG: Citrullinated peptide and its relevance to rheumatoid arthritis: An update. Int J Rheum Dis 13: 284-287, 2010.

12. Olson AL,Swigris JJ,SprungerDB,Fischer A,Fernandez-PerezER, Solomon J, Murphy J, Cohen M, Raghu G and Brown KK: Rheumatoid arthritis-interstitial lung disease-associated mortality. Am J Respir Crit Care Med 183: 372-378, 2011.

13. Kim HP, Wang X, Chen ZH, Lee SJ, Huang MH, Wang Y, Ryter SW and Choi AM: Autophagic proteins regulate cigarette smoke-induced apoptosis: Protective role of heme oxygenase-1. Autophagy 4: 887-895, 2008.

14. Cuervo AM, Bergamini E, Brunk UT, Dröge W, Ffrench M and Terman A: Autophagy and aging: The importance of maintaining 'clean' cells. Autophagy 1: 131-140, 2005.

15. Patel AS, Lin L, Geyer A, Haspel JA, An CH, Cao J, Rosas IO and Morse D: Autophagy in idiopathic pulmonary fibrosis. PLoS One 7: e41394, 2012.

16. Rangarajan S, Kurundkar A, Kurundkar D, Bernard K, Sanders YY, Ding Q, Antony VB, Zhang J, Zmijewski J and Thannickal VJ: Novel mechanisms for the antifibrotic action of Nintedanib. Am J Respir Cell Mol Biol 54: 51-59, 2016.

17. Shin YJ, Han SH, Kim DS, Lee GH, Yoo WH, Kang YM, Choi JY, Lee YC, Park SJ, Jeong SK, et al: Autophagy induction and CHOP under-expression promotes survival of fibroblasts from rheumatoid arthritis patients under endoplasmic reticulum stress. Arthritis Res Ther 12: R19, 2010.

18. Ireland JM and Unanue ER: Autophagy in antigen-presenting cells results in presentation of citrullinated peptides to CD4 T cells. J Exp Med 208: 2625-2632, 2011.

19. Samara KD, Trachalaki A, Tsitoura E, Koutsopoulos AV, Lagoudaki ED, Lasithiotaki I, Margaritopoulos G, Pantelidis P, Bibaki E, Siafakas NM, et al: Upregulation of citrullination pathway: From Autoimmune to Idiopathic Lung Fibrosis. Respir Res 18: 218, 2017.

20. Selman M and Pardo A: Revealing the pathogenic and aging-related mechanisms of the enigmatic idiopathic pulmonary fibrosis. an integral model. Am J Respir Crit Care Med 189: 1161-1172, 2014.

21. Wynn TA and Vannella KM: Macrophages in tissue repair, regeneration, and fibrosis. Immunity 44: 450-462, 2016.

22. Drakopanagiotakis F, Xifteri A, Tsiambas E, Karameris A, Tsakanika K, Karagiannidis N, Mermigkis D, Polychronopoulos V and Bouros D: Decreased apoptotic rate of alveolar macrophages of patients with idiopathic pulmonary fibrosis. Pulm Med 2012: 981730, 2012.

23. Larson-Casey JL, Deshane JS, Ryan AJ, Thannickal VJ and Carter AB: Macrophage Akt1 kinase-mediated mitophagy modulates apoptosis resistance and pulmonary fibrosis. Immunity 44: 582-596, 2016.

24. Antoniou KM,Margaritopoulos G,EconomidouFand Siafakas NM: Pivotal clinical dilemmas in collagen vascular diseases associated with interstitial lung involvement. Eur Respir J 33: 882-896, 2009. 
25. Lake F and Proudman S: Rheumatoid arthritis and lung disease: From mechanisms to a practical approach. Semin Respir Crit Care Med 35: 222-238, 2014.

26. Raghu G: Idiopathic pulmonary fibrosis: Guidelines for diagnosis and clinical management have advanced from consensus-based in 2000 to evidence-based in 2011. Eur Respir J 37: 743-746, 2011.

27. Arnett FC, Edworthy SM, Bloch DA, McShane DJ, Fries JF, Cooper NS, Healey LA, Kaplan SR, Liang MH, Luthra HS, et al: The American Rheumatism Association 1987 revised criteria for the classification of rheumatoid arthritis. Arthritis Rheum 31: 315-324, 1988.

28. Barth S, Glick D and Macleod KF: Autophagy: Assays and artifacts. J Pathol 221: 117-124, 2010.

29. Margaritopoulos GA, Tsitoura E, Tzanakis N, Spandidos DA, Siafakas NM, Sourvinos G and Antoniou KM: Self-eating: Friend or foe? The emerging role of autophagy in idiopathic pulmonary fibrosis. BioMed Res Int 2013: 420497, 2013.

30. Mora AL, Bueno M and Rojas M: Mitochondria in the spotlight of aging and idiopathic pulmonary fibrosis. J Clin Invest 127: 405-414, 2017.

31. Bueno M, Lai YC, Romero Y, Brands J, St Croix CM, Kamga C, Corey C, Herazo-Maya JD, Sembrat J, Lee JS, et al: PINK1 deficiency impairs mitochondrial homeostasis and promotes lung fibrosis. J Clin Invest 125: 521-538, 2015.

32. Kobayashi K, Araya J, Minagawa S, Hara H, Saito N, Kadota T, Sato N, Yoshida M, Tsubouchi K, Kurita Y, et al: Involvement of PARK2-mediated mitophagy in idiopathic pulmonary fibrosis pathogenesis. J Immunol 197: 504-516, 2016.

33. Park JH, Kim DS, Park IN, Jang SJ, Kitaichi M, Nicholson AG and Colby TV: Prognosis of fibrotic interstitial pneumonia: Idiopathic versus collagen vascular disease-related subtypes. Am J Respir Crit Care Med 175: 705-711, 2007.

34. Antoniou KM, Walsh SL, Hansell DM, Rubens MR, Marten K, Tennant R, Hansel T, Desai SR, Siafakas NM, du Bois RM, et al: Smoking-related emphysema is associated with idiopathic pulmonary fibrosis and rheumatoid lung. Respirology 18: 1191-1196, 2013.

35. Juge PA, Borie R, Kannengiesser C, Gazal S, Revy P, WemeauStervinou L, Debray MP, Ottaviani S, Marchand-Adam S, Nathan N, et al; FREX consortium: Shared genetic predisposition in rheumatoid arthritis-interstitial lung disease and familial pulmonary fibrosis. Eur Respir J 49: 49, 2017.

36. Araya J, Kojima J, Takasaka N, Ito S, Fujii S, Hara H, Yanagisawa H, Kobayashi K, Tsurushige C, Kawaishi M, et al: Insufficient autophagy in idiopathic pulmonary fibrosis. Am J Physiol Lung Cell Mol Physiol 304: L56-L69, 2013.
37. Kato M, Ospelt C, Gay RE, Gay S and Klein K: Dual role of autophagy in stress-induced cell death in rheumatoid arthritis synovial fibroblasts. Arthritis Rheumatol 66: 40-48, 2014.

38. Sorice M, Iannuccelli C, Manganelli V, Capozzi A, Alessandri C, Lococo E, Garofalo T, Di Franco M, Bombardieri M, Nerviani A, et al: Autophagy generates citrullinated peptides in human synoviocytes: A possible trigger for anti-citrullinated peptide antibodies. Rheumatology (Oxford) 55: 1374-1385, 2016.

39. Lee J, Hong EC, Jeong H, Hwang JW, Kim H, Bae EK, Ahn JK, Choi YL, Han J, Cha HS, et al: A novel histone deacetylase 6-selective inhibitor suppresses synovial inflammation and joint destruction in a collagen antibody-induced arthritis mouse model. Int J Rheum Dis 18: 514-523, 2015.

40. Rockel JS and Kapoor M: Autophagy: Controlling cell fate in rheumatic diseases. Nat Rev Rheumatol 12: 517-531, 2016.

41. Liang XH, Jackson S, Seaman M, Brown K, Kempkes B, Hibshoosh $\mathrm{H}$ and Levine B: Induction of autophagy and inhibition of tumorigenesis by beclin 1. Nature 402: 672-676, 1999 .

42. Semenza GL: Involvement of hypoxia-inducible factor 1 in pulmonary pathophysiology. Chest 128 (Suppl 6): 592S-594S, 2005.

43. Bellot G, Garcia-Medina R, Gounon P, Chiche J, Roux D, Pouysségur J and Mazure NM: Hypoxia-induced autophagy is mediated through hypoxia-inducible factor induction of BNIP3 and BNIP3L via their BH3 domains. Mol Cell Biol 29: 2570-2581, 2009.

44. Zhang H, Bosch-Marce M, Shimoda LA, Tan YS, Baek JH, Wesley JB, Gonzalez FJ and Semenza GL: Mitochondrial autophagy is an HIF-1-dependent adaptive metabolic response to hypoxia. J Biol Chem 283: 10892-10903, 2008

45. Ricci A, Cherubini E, Scozzi D, Pietrangeli V, Tabbì L, Raffa S, Leone L, Visco V, Torrisi MR, Bruno P, et al: Decreased expression of autophagic beclin 1 protein in idiopathic pulmonary fibrosis fibroblasts. J Cell Physiol 228: 1516-1524, 2013.

46. Sosulski ML, Gongora R, Danchuk S, Dong C, Luo F and Sanchez CG: Deregulation of selective autophagy during aging and pulmonary fibrosis: The role of TGF $\beta 1$. Aging Cell 14: 774-783, 2015.

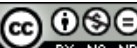

This work is licensed under a Creative Commons Attribution-NonCommercial-NoDerivatives 4.0 International (CC BY-NC-ND 4.0) License. 